Hautarzt 2018 $69: 298-305$

https://doi.org/10.1007/s00105-017-4119-9

Online publiziert: 1. Februar 2018

(c) Der/die Autor(en) 2018. Dieser Artikel ist eine Open-Access-Publikation.

CrossMark

\author{
B. Sachs ${ }^{1,5} \cdot$ T. Meier ${ }^{2} \cdot$ M. M. Nöthen ${ }^{3} \cdot$ C. Stieber ${ }^{3} \cdot$ J. Sting $\left.\right|^{4,5}$ \\ ${ }^{1}$ Klinik für Dermatologie und Allergologie, RWTH Aachen, Aachen, Deutschland \\ ${ }^{2}$ Abteilung Pharmakovigilanz, Bundesinstitut für Arzneimittel und Medizinprodukte, Bonn, Deutschland \\ ${ }^{3}$ Institut für Humangenetik, Universität Bonn, Bonn, Deutschland \\ ${ }^{4}$ Zentrum für Translationale Medizin, Universität Bonn, Bonn, Deutschland \\ ${ }^{5}$ Abteilung Forschung, Bundesinstitut für Arzneimittel und Medizinprodukte, Bonn, Deutschland
}

\title{
Arzneimittelassoziierte Angioödeme
}

\section{Bradykinin im Fokus}

\begin{abstract}
Das Auftreten von Angioödemen unter Therapie mit Angiotensinconverting-enzyme(ACE)-Hemmern ist bekannt und wird auf einen verminderten Bradykininabbau zurückgeführt. Auch für andere Arzneimittel wurde über ein erhöhtes Angioödemrisiko bei alleiniger Anwendung oder insbesondere in Kombination mit ACE-Hemmern berichtet. Als Ursache wurde bei einigen dieser Arzneimittel ebenfalls ein Zusammenhang mit Bradykinin vermutet. Ziel des Beitrags ist es, den Zusammenhang zwischen Arzneimittelanwendung als Mono- oder Kombinationstherapie und dem Auftreten bradykininmediierter Angioödeme zusammenfassend darzustellen.
\end{abstract}

\section{Angioödeme}

Angioödeme werden durch vaskuläre Reaktionen der tieferen Haut- und Schleim-

Der folgende Artikel basiert auf der Veröffentlichung Sachs B, Meier T (2017) Arzneimittel-assoziierte Angioödeme: Bradykinin im Fokus. Bulletin zur Arzneimittelsicherheit 2, S. 13-22. Die Recherche in der Nebenwirkungsdatenbank des Bundesinstituts für Arzneimittel und Medizinprodukte (BfArM) wurde aktualisiert (Infobox 1). Daher sind die Rechercheergebnisse in diesem Artikel nicht identisch mit den Rechercheergebnissen in dem Bezugsartikel [1].

Die in dem Artikel dargestellten Ansichten sind die der Autoren und nicht notwendigerweise die des Bundesinstitutes für Arzneimittel und Medizinprodukte. hautschichten verursacht, die mit einer lokalen Erweiterung der Blutgefäße und erhöhten Gefäßpermeabilität einhergehen und so zu einer Gewebeschwellung führen [2]. Typische Manifestationsorgane sind die Gesichtshaut, Schleimhäute von Zunge, Kehlkopf sowie v. a. bei hereditären Formen der Magen-Darm-Trakt [3]. Klinisch imponieren Angioödeme entsprechend ihrer Lokalisation mit zum Teil massiven prallelastischen Schwellungen, Atemnot (bei Lokalisation im Hals) oder unspezifischen Magen-DarmKrämpfen. Todesfälle durch Erstickungen kommen vor [3, 4]. Das klinische Erscheinungsbild „Angioödem“ "kann im Rahmen verschiedener Erkrankungen auftreten. In Bezug auf eine umfassende Klassifikation erworbener und hereditärer Angioödeme wird an dieser Stelle auf internationale Konsensuspublikationen verwiesen $[2,5]$. Auf klinisch praktischer Ebene ist eine Unterteilung in histaminvermittelte Angioödeme z. B. im Rahmen allergischer Reaktionen und bradykininvermittelte Angioödeme hilfreich [3].

\section{Histaminmediierte Angioödeme}

Histaminvermittelte Angioödeme entstehen durch eine Freisetzung von Histamin aus Mastzellen, das an Histaminrezeptoren bindet und so die Gefäßpermeabilität erhöht [7]. Die Histaminfreisetzung kann beispielsweise im Rahmen allergischer Reaktionen erfolgen, typischerweise vermittelt durch spezifisches
IgE (• Tab. 1; [7-9]). Klinisch treten diese Reaktionen in der Regel nicht isoliert, sondern zusammen mit anderen Symptomen, z.B. einer Urtikaria, auf [10], und es besteht Juckreiz [11]. Allerdings können histaminvermittelte Angioödeme als Symptom bei chronisch spontaner Urtikaria auch ohne Urtikaria auftreten [12]. Die Akuttherapie ist antiallergisch und erfolgt stadienorientiert, u. a. mit Gabe von Antihistaminika und Glukokortikoiden [7, 13-15].

\section{Bradykininmediierte Angioödeme}

Bradykininmediierte Angioödeme treten wesentlich seltener als histaminvermittelte Angioödeme auf [3, 16, 17]. Sie gehen klinisch nicht mit einer Urtikaria einher, ein Juckreiz fehlt deswegen typischerweise [11]. Sie können unterteilt werden in hereditäre oder erworbene Formen. Bei den hereditären Formen werden solche mit C1-Esteraseinhibitor(C1-INH)-Mangel (HAE [hereditäres Angioödem] Typ I) oder -Defekt (HAETyp II), verursacht durch Mutationen im SERPING1-Gen, von solchen mit normalem C1-Esteraseinhibitor (HAE Typ III, HAEnC1) unterschieden [3]. Bei einem Teil der HAEnC1-Patienten liegen Mutationen im Faktor-XII-Gen vor [18-20]. Bei erworbenen Formen kann als Folge von Erkrankungen (z. B. dem malignen Lymphom oder der monoklonalen Gammopathie) ebenfalls ein C1-INH-Mangel vorliegen [2, 3, 5]. Erworbene Formen mit normaler C1-INH- 


\section{Infobox 1 Hinweise zur Interpretation der Daten}

Die Ergebnisse ergeben sich aus der jeweiligen Recherchestrategie, die hier der Kürze halber nur auszugweise wiedergegeben wird: Inlandsfalle ohne zeitliche Begrenzung, Monosubstanz und Arzneimittelkombinationen, SMQ („Standardised MedDRA Queries“) „Angioödem", Stichtag 15.11.2017. Die Recherche schloss nur Suspected/interacting-Fälle ein, d.h. Fälle, in denen die gesuchte Monosubstanz/Arzneimittelkombination verdächtigt wurde, allein oder interagierend mit anderen Arzneimitteln das Angioödem ausgelöst zu haben, unabhängig davon, ob und ggf. welche anderen Arzneimittel gegeben wurden. Bei Alteplase bezieht sich das Ergebnis auf den ATC(anatomisch-therapeutisch-chemischesKlassifikationssystem)-Code B, bei Sirolimus auf den ATC-Code L.

Es handelt sich bei den Berichten sowohl um Spontanmeldungen als auch um Berichte aus systematisierten Untersuchungen, aus Projekten oder aus der Literatur. Die Meldungen sind dem Bundesinstitut für Arzneimittel und Medizinprodukte durch pharmazeutische Unternehmer, durch die Arzneimittelkommissionen der Heilberufe, direkt durch niedergelassene oder in Krankenhäusern tätige Arzte sowie durch sonstige Meldequellen, z. B. von Patienten, berichtet worden. Es muss beachtet werden, dass es sich um Verdachtsfälle unerwünschter Arzneimittelwirkungen handelt, dass also ein Kausalzusammenhang im Einzelfall nicht sicher belegt ist. Ferner lassen sich auf der Grundlage von Spontanberichten keine Aussagen darüber machen, wie häufig eine bestimmte unerwünschte Wirkung unter der Anwendung eines Arzneimittels vorkommt. Auch vergleichende Angaben darüber, wievielmal häufiger eine bestimmte unerwünschte Wirkung bei einem Arzneimittel im Verhältnis zu einem anderen Arzneimittel auftritt, sind anhand solcher Berichte kaum möglich.

Aktivität treten bei Einnahme exogener Substanzen, wie z.B. ACE-Hemmern, auf (• Tab. 1; [5, 11]).

In der pathophysiologischen Endstrecke kommt es typischerweise $\mathrm{zu}$ einer erhöhten Bradykininkonzentration entweder als Folge einer gesteigerten Produktion (z. B. bei bestimmten hereditären Formen) oder eines gestörten Bradykininabbaus (z. B. bei arzneimittelassoziierten Formen; [3, 21-23]). Dieser Beitrag fokussiert auf arzneimittelassoziierte, bradykininmediierte Angioödeme. Ein Prototyp dieser Reaktionen ist das ACE-Hemmer-induzierte Angioödem. Da dieser Angioödemtyp nicht durch eine Histaminausschüttung ausgelöst wird, ist eine antiallergische Akuttherapie mit Antihistaminika (aber auch mit Glukokortikoiden oder Adrenalin) in der Regel nicht wirksam [11, 21, 24]. Vielmehr umfassen die Therapieempfehlungen das Absetzen des ACE-Hemmers und gof. supportive Maßnahmen zur Offenhaltung der Luftwege [15]. In der Literatur werden als pharmakologische Möglichkeiten C1-INH und Icatibant, von einigen Autoren zusätzlich auch Fresh-Frozen-Plasma genannt $[11,21$, 23, 24]. Alle genannten Stoffe besitzen für die Indikation des ACE-Hemmerinduzierten Angioödems keine Zulassung.

\section{Kallikrein-Bradykinin-System}

\section{Entstehung und Degradierung von Bradykinin}

Für eine umfassende Darstellung des komplexen Kallikrein-Bradykinin-Systems wird auf die entsprechende Literatur verwiesen. Prinzipiell erfolgt die Generierung von Bradykinin im Kallikrein-Bradykinin-System über einen intrinsischen (Faktor-XII-abhängigen) und einen extrinsischen Weg ( $\bullet$ Abb. 1; [24]). Der intrinsische Weg scheint, basierend auf Tierdaten, für die Aufrechterhaltung der Bradykiningrundspiegel von Bedeutung $\mathrm{zu}$ sein [24] und wird von einigen Autoren als hauptsächlicher Entstehungsweg angeführt [10].

》) Als wesentlicher Abbauweg
gilt die Degradierung von
Bradykinin zu inaktiven
Metaboliten

Bradykinin selbst hat eine sehr kurze Halbwertszeit (<30 s; [28]), da es schnell von ubiquitären Kininasen degradiert wird (• Abb. 1). Als wesentlicher Abbauweg wird die Degradierung von Bradykinin zu inaktiven Metaboliten beschrieben. Diese Degradierung wird v. a. vom $\mathrm{ACE}$ und zu einem geringeren
Teil durch die Aminopeptidase P (APP) katalysiert. Die neutrale Endopeptidase (NEP, auch Neprilysin genannt) und Dipeptidylpeptidase (DPP)-IV tragen noch einmal deutlich weniger zur Degradierung bei $(\mathrm{ACE}>\mathrm{APP}>>\mathrm{NEP}$, DPP$4 ;[23,26,29,30])$.

$\mathrm{Zu}$ einem sehr geringen Teil (ca. $<10 \%)$ wird Bradykinin durch Carboxypeptidasen zu dem aktiven Metaboliten des Arginin-9-Bradykinin metabolisiert [23, 28, 31, 32]. Dieser aktive Metabolit wird primär durch APP und durch ACE inaktiviert [23-25, 29, 31, 33].

\section{Rezeptoren und Wirkung}

Die Wirkung von Bradykinin wird über Rezeptoren vermittelt. Bis jetzt wurden 2 Rezeptoren (BKR-1 und BKR-2) identifiziert [21]. BKR-2 wird konstitutiv exprimiert, BKR-1 nur bei Entzündungszuständen, dann aber innerhalb von Stunden $[21,24,28]$.

Die Bindung von Bradykinin an BKR2 auf endothelialen Zellen führt zu Vasodilatation und verstärkter vaskulärer Permeabilität. Dies kann wiederum eine interstitielle Flüssigkeitsakkumulation und letztendlich ein Angioödem auslösen. Eine Aktivierung von BKR-1 führt ebenfalls zu Gefäßerweiterung und verstärkter Gefäßpermeabilität [11, 21].

Der aktive Metabolit von Bradykinin wirkt v. a. am BKR-1, während Bradykinin v. a. BKR-2 aktiviert und keine signifikante Aktivität für BKR-1 aufweist [23].

Bradykinin stimuliert auch die Freisetzung von Substanz $P$ aus sensorischen Nerven. Substanz P wiederum erhöht die Gefäßpermeabilität über Bindung an Neurokinin-1-Rezeptoren [23, 25, 27]. Substanz P wird durch ACE, NEP und DPP-IV degradiert $[23,27]$. Bei einer Hemmung von ACE erfolgt die Degradierung primär durch DPP-IV $[25,34$, 35]. 


\section{Arzneimittelassoziierte, bradykininmediierte Angioödeme}

\section{ACE-Hemmer}

Etwa 0,1-0,7\% der mit ACE-Hemmern behandelten Patienten entwickeln ein Angioödem [21, 36]. In der Nebenwirkungsdatenbank des BfArM liegen rund 2000 Meldungen über Angioödeme im Zusammenhang mit der Anwendung von ACE-Hemmern in Deutschland vor (-Infobox 1). Das zeitliche Spektrum des Auftretens von Angioödemen nach Anwendung von ACE-Hemmern reicht vom ersten Anwendungstag bis $\mathrm{zu}$ Manifestationen nach jahrelanger Anwendung. Etwa zwei Drittel der Angioödeme treten innerhalb der ersten 3 Monate auf [4, 37, 38]. Häufig betreffen sie das Gesicht, die Lippen oder die Zunge [39] und verlaufen mild, also ohne eine Verlegung der Atemwege [11]. Allerdings wurden auch Todesfälle beschrieben [40]. Aufgrund der hohen Anwendungshäufigkeit von ACE-Hemmern wird weltweit mit mehreren 100 tödlichen Verläufen pro Jahr gerechnet [41].

\section{Pathophysiologie ACE-Hemmer- induzierter Angioödeme}

Beim ACE-Hemmer-induzierten Angioödem ist der Bradykininabbau gestört [42], und es kann zu einer Verdopplung des Bradykininspiegels im Blut kommen $[26,43]$. Es wird davon ausgegangen, dass in der Situation einer Hemmung des ACE durch die Einnahme von ACEHemmern die Degradierung von Bradykinin zu inaktiven Metaboliten nun stärker von den anderen Enzymen, nämlich APP, NEP und DPP-IV, abhängt $[24,25]$, wobei APP den größten Beitrag leistet [23, 34]. Weiterhin soll die im physiologischen Zustand untergeordnete Metabolisierung von Bradykinin über Carboxypeptidasen zu dem aktiven Metaboliten an Bedeutung gewinnen. Entsprechend ist die Degradierung von Bradykinin durch Carboxypeptidasen im Serum von Patienten mit ACEHemmer-induziertem Angioödem im Vergleich zu Kontrollpersonen erhöht

Hautarzt 2018 -69:298-305 https://doi.org/10.1007/s00105-017-4119-9

(c) Der/die Autor(en) 2018. Dieser Artikel ist eine Open-Access-Publikation.

\section{B. Sachs · T. Meier · M. M. Nöthen · C. Stieber · J. Stingl}

\section{Arzneimittelassoziierte Angioödeme. Bradykinin im Fokus}

\section{Zusammenfassung}

Bei Angioödemen können pathophysiologisch histamin- und bradykininmediierte Formen unterschieden werden. Angiotensin-converting-enzyme(ACE)-Hemmerinduzierte Angioödeme sind der Prototyp arzneimittelassoziierter, bradykininmediierter Angioödeme. Als Ursache wird ein verminderter Bradykininabbau durch die Hemmung des ACE angenommen. In diesem Fall kommt anderen Bradykinin degradierenden Enzymen eine größere Bedeutung zu. Wenn auch diese z. B. durch genetische Varianten oder äußere Faktoren in ihrer Wirkung vermindert sind, kann die Hemmung des ACE möglicherweise nicht ausreichend kompensiert werden. Auch für andere Arzneimittel wurde über ein erhöhtes Angioödemrisiko bei alleiniger Anwendung oder insbesondere in Kombination mit ACE-Hemmern berichtet. Ursächlich wurde ebenfalls ein Zusammenhang mit dem Abbau von Bradykinin vermutet. Dabei könnten bei den Angioödemen, die bei gleichzeitiger Anwendung von ACE-Hemmern mit anderen Arzneimitteln auftreten, additive Effekte in Bezug auf den Bradykininabbau eine Rolle spielen.

\section{Schlüsselwörter}

Angiotensin-converting-enzyme-Hemmer . Angiotensinrezeptorblocker - Sartane Nebenwirkung $\cdot$ Aminopeptidase P

\section{Drug-induced angioedema. Focus on bradykinin}

\section{Abstract}

On a pathophysiological level, angioedema can be differentiated into histamine- and bradykinin-mediated types. The prototype drug-associated, bradykinin-mediated form of angioedema is angiotensin-converting enzyme (ACE) inhibitor-induced angioedema. The hypothesized cause is a decrease in bradykinin degradation via ACE inhibition. In this scenario, other bradykinin-degrading enzymes assume major importance. When the effect of these enzymes is also diminished, e. g., due to genetic variants or external factors, compensation for the inhibition of ACE may be insufficient. An increased risk of angioedema has also been reported for other drugs, particularly when prescribed in combination with ACE inhibitors. Here, the suspected cause also relates to the degradation of bradykinin. When angioedema arises within the context of concomitant ACE inhibitor use, additive bradykinin degradation effects may be implicated.

\section{Keywords}

Angiotensin-converting enzyme inhibitors . Angiotensin receptor blockers - Sartans - Side effect $\cdot$ Aminopeptidase $P$
[25, 44]. Wenn allerdings die Aktivität dieser kompensatorisch tätigen Enzyme aufgrund von Komedikationen oder genetischer Varianten vermindert ist, kann die Hemmung des ACE durch ACEHemmer trotz redundanter Abbauwege möglicherweise nicht ausreichend kompensiert werden [2, 23, 45].

\section{Bedeutung kompensatorisch tätiger Enzyme}

\section{Aminopeptidase $\mathbf{P}$}

Bei europäischen Patienten mit ACEHemmer-induziertem Angioödem wurden eine verminderte APP-Aktivität und Degradierung des aktiven Metaboliten im Serum festgestellt $[45,46]$. Weiterhin wurden in mehreren Studien genetische Assoziationen beschrieben, die mit einer reduzierten APP-Aktivität und erhöhten Inzidenz für ACE-Hemmer-induzierte Angioödeme einhergehen [23, 29, 31, 47]. In einer Studie wurde beispielsweise eine Odds Ratio (OR) von 4,87 (95\%Konfidenzintervall [KI]: 1,78-13,35, $p=0,002)$ für einen funktionalen Polymorphismus, der über eine verminderte Plasmaaktivität von APP mit einer verminderten Metabolisierung von Bradykinin verbunden sein soll, berichtet [29]. Interessanterweise wurde auch ein nur in Personen schwarzafrikanischer Herkunft vorkommender Polymorphismus, 
Tab. 1 Klassifizierung von Angioödemen. (Mod. nach Lang et al. [2] und Cicardi et al. [5])

Klassifikation Bradykininvermittelte Angioödeme

C1-INH-Mangel/Defekt

\begin{tabular}{|c|c|c|c|c|c|c|c|}
\hline Eigenschaften & Vererbt & Erworben & Vererbt & Erworben & $\begin{array}{l}\text { IgE- } \\
\text { mediiert }\end{array}$ & $\begin{array}{l}\text { Nicht-IgE-medi- } \\
\text { iert }\end{array}$ & Erworben \\
\hline \multirow[t]{2}{*}{ Krankheit } & $\begin{array}{l}\text { HAE-I } \\
\text { C1-INH-Plasma- } \\
\text { konzentration } \downarrow \\
\text { Aktivität } \downarrow\end{array}$ & $\begin{array}{l}\text { Verbrauch von C1-INH } \\
\text { durch lympho- } \\
\text { proliferative/ } \\
\text { Autoimmunerkrankungen }\end{array}$ & $\begin{array}{l}\text { FXII-HAE } \\
\text { Spezifische } \\
\text { Mutation } \\
\text { im FXII-Gen } \\
\text { nachweisbar }\end{array}$ & $\begin{array}{l}\text { Arzneimittel- } \\
\text { induziert, z. B. } \\
\text { ACE-Hemmer }\end{array}$ & $\begin{array}{l}\text { Allergische } \\
\text { Reaktion }\end{array}$ & $\begin{array}{l}\text { Chronische } \\
\text { spontane/ } \\
\text { induzierbare } \\
\text { Urtikaria, nicht } \\
\text { klassifiziertes AE }\end{array}$ & $\begin{array}{l}\text { Nicht klassifi- } \\
\text { ziertes } A E\end{array}$ \\
\hline & $\begin{array}{l}\text { HAE-II } \\
\text { C1-INH-Funktion } \\
\text { gestört } \\
\text { C1-INH-Plasma- } \\
\text { konzentration } \\
\text { normal oder } \uparrow\end{array}$ & $\begin{array}{l}\text { Autoantikörper gegen } \\
\text { C1-INH }\end{array}$ & $\begin{array}{l}\text { U-HAE } \\
\text { Unbekannter } \\
\text { genetischer } \\
\text { Defekt }\end{array}$ & - & - & - & - \\
\hline $\begin{array}{l}\text { Auftreten von } \\
\text { Quaddeln }\end{array}$ & Nein & Nein & Nein & Nein & Ja/nein & Ja/nein & Nein \\
\hline
\end{tabular}

vermittelte Angioödeme

Normale C1-INH-Funktion

thische An-

gioödeme

Normale C1-INH-Funktion

Normale

C1-INH-

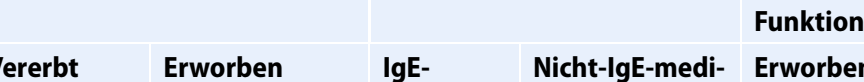

mediiert iert

lassifiziertes $A$

$\mathrm{U}-\mathrm{HAE}$

Defekt
Aktivität während eines Angioödems reduziert ist. Dies sei nicht der Fall, wenn das Angioödem abgeklungen ist oder wenn ACE-Hemmer eingenommen werden, ohne dass ein Angioödem auftritt. Weiterhin sollen erhöhte DPPIV-Spiegel mit erhöhten Blutzuckerspiegeln einhergehen und Diabetiker daher ein niedrigeres Risiko für das Auftreten eines ACE-Hemmer-induzierten Angioödems im Vergleich zu Nichtdiabetikern haben (RR: 0,88, $95 \%$-KI: 0,82-0,95; [36]). Andererseits sei die DPP-IV-Aktivität bei Rauchern erniedrigt, was zu der Beobachtung passt, dass für Raucher ein höheres Risiko für Angioödeme unter ACE-Hemmern beschrieben wurde (s.oben; [11, 23, 52]).

\section{ACE-Hemmer-induzierte Angioödeme - multifaktoriell verursacht}

Zusammenfassend ist beim Entstehen ACE-Hemmer-induzierter Angioödeme von einer multifaktoriellen Genese unter Beteiligung genetischer und äußerer Einflussfaktoren auszugehen. Möglicherweise gibt es auch verschiedene Untergruppen, bei denen z. B. jeweils andere, kompensatorisch in die Degradierung von Bradykinin und/oder Substanz $\mathrm{P}$ involvierte Enzyme, genetisch bedingt oder aufgrund äußerer Einflussfaktoren, weniger wirksam sind. [23].

Bisher ist erst eine großflächige genetische Untersuchung veröffentlicht worden, in der genomweit genetische Varianten (genomweite Assoziationsstudie [GWAS]) untersucht wurden [47]. Dabei zeigte sich in einer begrenzten Stichprobe (175 Patienten und 489 Kontrollen) kein genomweit signifikanter Befund. Das deutet darauf hin, dass eine größere Zahl von Genen beteiligt ist. In zukünftigen, größeren Stichproben wird man diese identifizieren können und damit erstmals einen umfassenden Einblick in die beteiligten biologischen Mechanismen erhalten. Es wird interessant sein, inwieweit Faktoren identifiziert werden, die über den Stoffwechselweg des Bradykininabbaus hinausgehen. So fanden sich in der erwähnten ersten GWAS unter der Schwelle genomweiter Signifikanz Hinweise auf eine Beteiligung immunregulatorischer Gene [47].

\section{Angiotensinrezeptorblocker}

Auch unter Anwendung von Angiotensinrezeptorblockern (ARB) wurden Angioödeme beobachtet. Sie sollen etwas weniger als halb so häufig vorkommen thophysiologische Bedeutung hat. Dafur
spricht aus ihrer Sicht, dass die DPP-IV- 


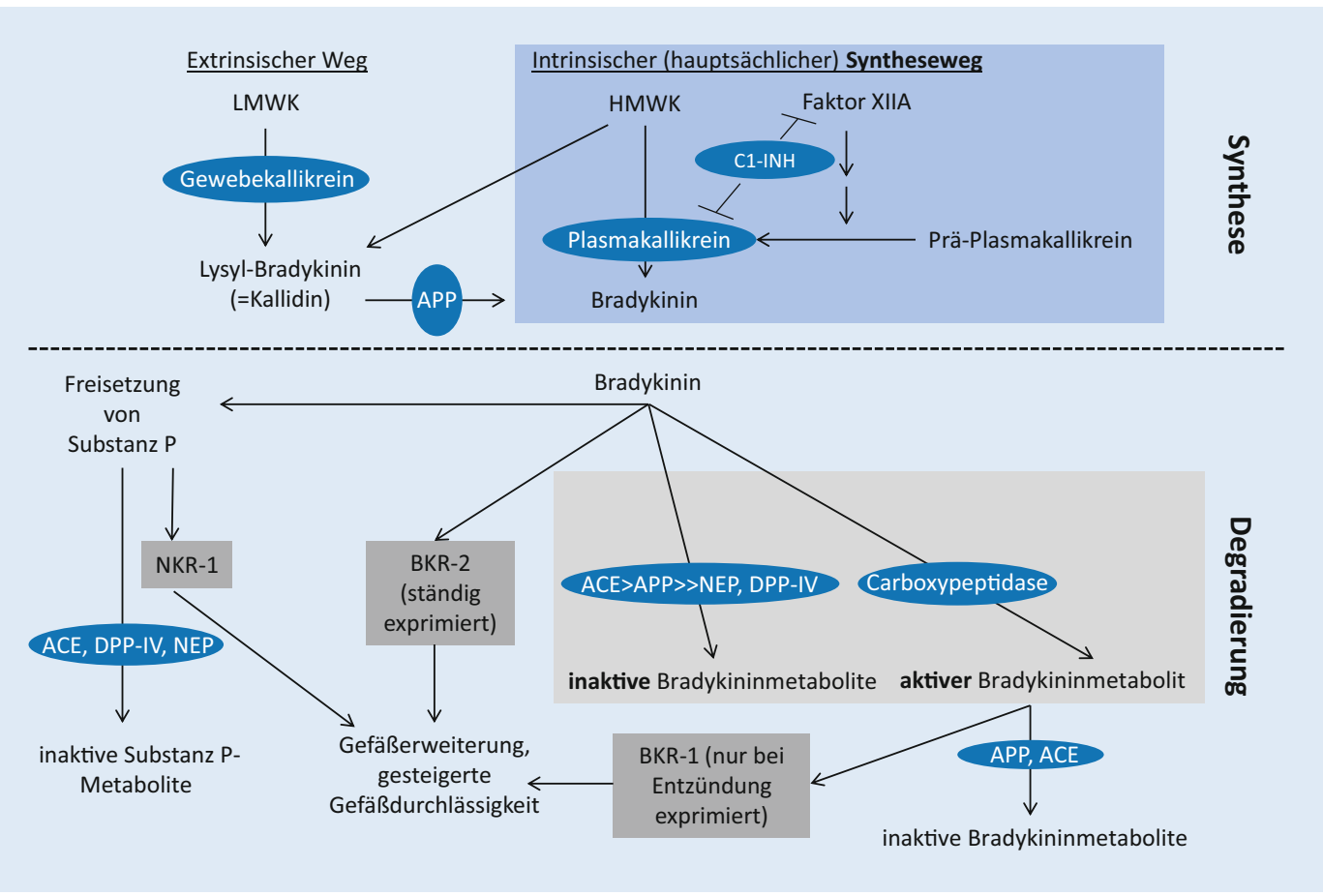

Abb. $1<$ Vereinfachende Darstellung der diskutierten Synthese- und Degradierungswege sowie der Wirkungsvermittlung von Bradykinin. Nähere Beschreibung s. Text. $A C E_{\text {" }}$ angiotensin-converting enzyme", APP Aminopeptidase $\mathrm{P}, L M W K$, Iow molecular weight kininogen", HMWK "high molecular weight kininogen", BKR-1/2 Bradykininrezeptor $1 / 2$, NKR-1 Neurokininrezeptor 1,NEP neutrale Endopeptidase, DPP-IVDipeptidylpeptidase IV, C1-INHC1-Esteraseinhibitor hemmt. (Nach Bezalel et al. [11], Bork [22], Byrd et. al [25], Campbell [26]; Hoover et al. [23], Kaplan [24], Levy et al. [10], Scott et al. [27])

wie unter ACE-Hemmern [11, 21]. In der Nebenwirkungsdatenbank des BfArM liegen rund 820 Meldungen über Angioödeme im Zusammenhang mit der Anwendung von ARB vor (•Infobox 1).

ARB sollen den Blutdruck ausschließlich dadurch senken, dass sie die Bindung von Angiotensin II an seinem Rezeptor blockieren, aber keinen Einfluss auf die Aktivität von ACE haben und die Degradierung von Bradykinin nicht inhibieren [11]. Andererseits weist eine Studie darauf hin, dass ARB auch in das Bradykininsystem eingreifen könnten, da ihre gefäßerweiternde Wirkung durch den Bradykininrezeptorantagonisten Icatibant gehemmt werden kann [53]. Für einen Vertreter der ARB wurde gezeigt, dass die Bradykininspiegel im Plasma von Patienten mit Hypertonie ungefähr verdoppelt werden [54]. Es wurde vermutet, dass durch $\mathrm{ARB}$ ausgelöste Angioödeme mit einer verminderten Degradierung von Bradykinin durch ACE und NEP im Zusammenhang stehen [54]. Patienten, die unter ACE-Hemmern ein Angioödem entwickelten, haben ein moderates Risiko (2-17\%) für das Auftreten eines erneuten Angioödems, wenn sie auf ARB umgestellt werden $[55,56]$.

\section{Racecadotril}

Racecadotril ist zur Behandlung des akuten Durchfalls, wenn eine ursächliche Therapie nicht möglich ist, zugelassen. Dabei ist Racecadotril die Vorstufe zu dem eigentlichen Wirkstoff Thiorphan. Thiorphan wiederum ist ein selektiver NEP-Hemmer. Da auch Bradykinin ein Substrat der NEP ist, könnte durch Hemmung des Bradykininabbaus die Entstehung von Angioödemen ausgelöst werden.

Tatsächlich sind Angioödeme ein bekanntes Risiko bei Anwendung von Racecadotril. Allerdings scheinen sie wesentlich seltener aufzutreten als bei mit ACE-Hemmern behandelten Patienten. Dies entspricht auch den Ergebnissen einer Studie an Arteriolen von Patienten mit Arteriosklerose, die zeigte, dass Thiorphan weniger potent als der ACEHemmer den Bradykininmetabolismus hemmt [57]. In einer neueren Analyse der französischen Nebenwirkungsdatenbank zu bradykininmediierten Angioödemen fand sich keine Meldung zum Auftreten eines Angioödems unter Racecadotril [17]. In der Nebenwirkungsdatenbank des BfArM liegen rund $20 \mathrm{Mel}-$ dungen über Angioödeme im Zusam- menhang mit der Anwendung von Racecadotril vor (•Infobox 1$)$.

Angioödeme, die in Spontanberichten gemeldet wurden, betrafen in erster Linie das Gesicht und die Mundhöhle inklusive Schwellungen von Lippen und Zunge. Nur selten wurde zusätzlich Urtikaria berichtet. Dies passt zu der Vorstellung eines bradykininmediierten Geschehens. Aus den Spontanberichten geht hervor, dass die Mehrheit der Angioödeme unter Racecadotril bei Patienten auftrat, die nicht zeitgleich mit einem ACE-Hemmer behandelt wurden. Eine ACE-Hemmung scheint daher keine Voraussetzung für die Entstehung eines Angioödems unter Anwendung von Racecadotril zu sein.

Im Gegensatz zu den Spontanberichten, die über Angioödeme bei alleiniger Anwendung von Racecadotril informieren, steht eine Tierstudie an Ratten, die darauf hindeutet, dass eine NEP-Hemmung allein keinen vasodilatatorischen und die Gefäßpermeabilität steigernden Effekt hat, dass aber ein ACE-Hemmer, zusammen mit einem NEP-Hemmer gegeben, in Bezug auf die Entstehung eines Angioödems additiv wirkt [58]. 


\section{Gewebeplasminogenaktivator}

Das Auftreten von Angioödemen unter Anwendung von Gewebe(„tissue“)Plasminogenaktivator (tPA; Alteplase) bei Patienten mit ischämischem Schlaganfall ist bekannt [59]. Die Häufigkeit soll ca. 1,3-5,9\%, nach einer aktuelleren Publikation von 2015 sogar 7,9\% betragen (42/530 Patienten, 95\%-KI: 5,5-10,6; [59]). In dieser retrospektiven Analyse wurde darüber hinaus gezeigt, dass tPA-assoziierte Angioödeme mit der vorherigen Einnahme von ACE-Inhibitoren assoziiert sind (OR: 2,3, $95 \%$ KI: 1,1-4,7; [59]). In einer älteren Studie war diesbezüglich ein höheres Risiko beschrieben worden (OR: 16,7, $95 \%$-KI: 3,3-84,3; [60]).

In der Nebenwirkungsdatenbank des BfArM liegen rund 130 Meldungen über Angioödeme im Zusammenhang mit der Anwendung von Alteplase vor (• Infobox 1). Plasminogen wird von tPA zu Plasmin hydrolysiert, das wiederum hochmolekulares Kininogen spaltet und dadurch Bradykinin freisetzt. In Bezug auf die Pathophysiologie wurde spekuliert, dass tPA die Bradykininbildung durch diesen Mechanismus steigert [59]. Daher könnte theoretisch sowohl eine erhöhte Produktion von Bradykinin durch tPA als auch eine verminderte Degradierung von Bradykinin (durch ACEHemmer) das Risiko für Angioödeme erhöhen [60]. Dadurch ließe sich das erhöhte Risiko für tPA-assoziierte Angioödeme unter gleichzeitiger Therapie mit ACE-Hemmern erklären.

\section{Dipeptidylpeptidase-IV-Hemmer}

DPP-IV ist ein multifunktionales Enzym, das eine Rolle u. a. im Immunsystem und der Regulierung des Blutzuckerspiegels spielt $[25,61]$. Es degradiert „glucagonlike peptide I“ (GLP-1; [34, 42]). Über eine Hemmung von DPP-IV verlängern Gliptine (DPP-IV-Hemmer) die GLP-1abhängige Insulinsekretion in den Betazellen des Pankreas und werden daher zur oralen Therapie des Typ-2-Diabetes eingesetzt [34, 62, 63]. DPP-IV degradiert allerdings auch Bradykinin und Substanz P [23, 25-27, 30]. In einer Metaanalyse wurde gezeigt, dass die Anwen- dung von Vildagliptin als ein Vertreter der Gliptine nicht mit einem erhöhten Angioödemrisiko verbunden ist [34]. Allerdings war das Risiko bei Patienten, die gleichzeitig ACE-Hemmer anwendeten, erhöht (OR: 4,57, 95\%-KI: 1,57-13,28; [34]). In der Literatur wird aber auch vermutet, dass DPP-IV-Hemmer-assoziierte Angioödeme weniger häufig berichtet werden, da im klinischen Alltag v. a. ACE-Hemmer-induzierte Angioödeme präsent sind [27]. In der $\mathrm{Ne}$ benwirkungsdatenbank des BfArM liegen rund 140 Meldungen über Angioödeme im Zusammenhang mit der Anwendung von Gliptinen vor (• Infobox 1$)$.

\section{mTOR-Hemmer}

Als mTOR-Hemmer (mTOR: „mechanistic [or mammalian] target of rapamycin") wird eine Gruppe von Substanzen beschrieben, die die mTOR-Signalwege (mTORC1 und mTORC2) unterbrechen [64]. Bekannte Vertreter sind beispielsweise Sirolimus und Everolimus, die u. a. zur Immunsuppression z. B. bei Transplantatempfängern eingesetzt werden [64].

In der Nebenwirkungsdatenbank des BfArM liegen rund 370 Meldungen über Angioödeme im Zusammenhang mit der Anwendung von Everolimus und rund 40 Meldungen zu Sirolimus vor (•Infobox 1). Eine erhöhte Inzidenz von Angioödemen bei Organtransplantatempfängern unter Behandlung mit mTOR-Hemmern wurde in Fallberichten beschrieben [65]. In einer Querschnittsstudie traten bei 12 von 80 (15\%) nierentransplantierten $\mathrm{Pa}$ tienten unter Therapie mit Sirolimus Angioödeme ohne Urtikaria auf [66]. Die Autoren vermuteten eine kausale Rolle von Sirolimus bei der Entstehung der Angioödeme. Allerdings nahmen von den 12 Patienten 4 ACE-Hemmer, 2 ARB und einer einen ACE-Hemmer zusammen mit Tacrolimus ein [66].

In einer retrospektiven Analyse nahmen von 309 nierentransplantierten Patienten, die mTOR-Hemmer erhalten hatten, 137 zusätzlich ACE-Hemmer ein [65]. Diese Kombinationsbehandlung war mit einer Angioödeminzidenz von $6,6 \%$ vs. $1,9 \%$ bei alleiniger Therapie mit mTOR-Hemmern verbunden [65]. Generell scheinen Angioödeme nach Angaben der Autoren bei mit Sirolimus behandelten Patienten mild zu verlaufen [65].

Es wurde spekuliert, dass Immunsuppressiva das Risiko für ACE-Hemmerinduzierte Angioödeme erhöhen, indem sie die Aktivität von DPP-IV reduzieren [67]. Dieser Überlegung liegen die Beobachtungen zugrunde, dass DPP-IV (auch als Oberflächenmarker CD26 bekannt) eine Rolle bei der Aktivierung und Proliferation von T-Lymphozyten spielt [61, 67]. Immunsuppressiva erniedrigen die Expression von CD26 auf T-Lymphozyten und die Aktivität des zirkulierenden DPP-IV [67]. Diese Befunde passen zu der Beobachtung, dass eine Assoziation eines reduzierten DPP-IV-Spiegels unter Immunsuppressiva mit einem Anstieg von Angioödemen bei Patienten beobachtet wurde, die gleichzeitig ACEHemmer einnahmen [67].

\section{Große bevölkerungsbezogene Relevanz}

Die Verordnung von ACE-Hemmern zulasten der gesetzlichen Krankenkassen in Deutschland belief sich 2016 auf rund 5700 Mio. DDD („defined daily dose“ [definierte Tagesdosen]), die für $\mathrm{ARB}$ auf rund 3000 Mio. DDD und die für Gliptine auf rund 360 Mio. DDD (Monound Kombinationsarzneimittel; [68]). In der Literatur wird für Deutschland eine Inzidenz von 20.000 bis 35.000 Fällen von ACE-Hemmer-induzierten Angioödemen pro Jahr geschätzt [21].

\section{》) In Deutschland kommt es geschätzt jährlich zu 20.000 bis 35.000 Fällen von ACE-Hemmer- induzierten Angioödemen}

Auch für andere Arzneimittel wurde über ein erhöhtes Risiko für bradykininmediierte Angioödeme bei alleiniger Anwendung berichtet. Darüber hinaus scheinen bestimmte Arzneimittel bei gleichzeitiger Anwendung mit ACE-Hemmern das Risiko durch additive Effekte auf den Bradykininabbau weiter erhöhen zu können. 
Wie in dem Beitrag dargestellt, kann es individuelle Variabilitäten im Bradykininabbau geben, entweder genetisch oder durch äußere Faktoren bedingt, die die Empfänglichkeit für ACE-Hemmer-induzierte Angioödeme erhöhen könnten. Insbesondere große systematische genetische Untersuchungen stehen allerdings noch aus und werden möglichweise völlig neue Einblicke in die beteiligten biologischen Zusammenhänge ermöglichen.

Aufgrund der großen bevölkerungsbezogenen Relevanz geht das BfArM dieser Thematik zusammen mit dem Institut für Humangenetik der Universität Bonn in einem aktuellen Forschungsprojekt weiter nach. Abschließend soll mit diesem Beitrag auch auf die Möglichkeit aufmerksam gemacht werden, an dieser Studie durch Rekrutierung von Patienten teilzunehmen.

\section{Fazit für die Praxis}

- Klinisch unterscheiden sich bradykinin- von histaminmediierten Angioödemen u.a. durch den fehlenden Juckreiz.

- ACE-Hemmer-induzierte Angioödeme sind der Prototyp arzneimittelassoziierter, bradykininmediierter Angioödeme. In der Literatur wird für Deutschland aufgrund der hohen Anwendungszahlen von ACE-Hemmern eine Inzidenz von $\mathbf{2 0 . 0 0 0}$ bis $\mathbf{3 5 . 0 0 0}$ Fällen pro Jahr geschätzt

- Etwa zwei Drittel der ACE-Hemmerinduzierten Angioödeme treten innerhalb der 3 ersten Anwendungsmonate auf, sie können sich aber auch erst nach jahrelanger Anwendung manifestieren.

- Therapeutisch ist eine antiallergische Therapie in der Regel nicht hilfreich. Die Therapieempfehlungen umfassen das Absetzen des ACE-Hemmers und ggf. supportive Maßnahmen zur Offenhaltung der Luftwege.

- Auch für andere Arzneimittel wurde über ein erhöhtes Angioödemrisiko bei alleiniger Anwendung oder in Kombination mit ACE-Hemmern berichtet. Ursächlich wurde ebenfalls ein Zusammenhang mit dem Abbau von Bradykinin vermutet.

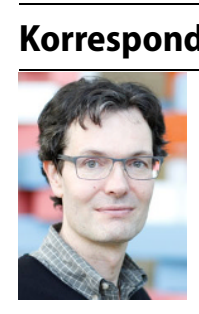

\section{Korrespondenzadresse}

Prof. Dr. B. Sachs

Abteilung Forschung,

Bundesinstitut für

Arzneimittel und

Medizinprodukte

Kurt-Georg-Kiesinger-Allee 3, 53175 Bonn, Deutschland Bernhardt.sachs@bfarm.de

\section{Einhaltung ethischer Richtlinien}

Interessenkonflikt. B. Sachs, T. Meier, M.M. Nöthen und J. Stingl geben an, dass kein Interessenkonflikt besteht. C. Stieber erhielt von der Firma Shire Reisekostenunterstützung und ein Honorar für eine Tätigkeit in einem Advisory Board.

Dieser Beitrag beinhaltet keine von den Autoren durchgeführten Studien an Menschen oder Tieren.

Open Access. Dieser Artikel wird unter der Creative Commons Namensnennung 4.0 International Lizenz (http://creativecommons.org/licenses/by/4.0/deed. de) veröffentlicht, welche die Nutzung, Vervielfältigung, Bearbeitung, Verbreitung und Wiedergabe in jeglichem Medium und Format erlaubt, sofern Sie den/die ursprünglichen Autor(en) und die Quelle ordnungsgemäßnennen, einen Link zur Creative Commons Lizenz beifügen und angeben, ob Änderungen vorgenommen wurden.

\section{Literatur}

1. Sachs B, Meier T (2017) Arzneimittel-assoziierte Angioödeme: Bradykinin im Fokus. Bulletin zur Arzneimittelsicherheit 2, S13-22

2. Lang DM, Aberer W, Bernstein JA et al (2012) International consensus on hereditary and acquired angioedema. Ann Allergy Asthma Immunol 109:395-402

3. Bork K (2012) Hereditäres Angioödem durch C1Inhibitor-Mangel. Allergo J 21:109

4. Beltrami L, Zingale LC, Carugo S et al (2006) Angiotensin-converting enzyme inhibitor-related angioedema: how to deal with it. Expert Opin Drug Saf 5:643-649

5. Cicardi M, Aberer W, Banerji A et al (2014) Classification, diagnosis, and approach to treatment for angioedema: consensus report from the Here ditary Angioedema International Working Group. Allergy 69:602-616

6. Hellebrand M-C, Kojda G, Hoffmann TK, Bas M (2006) Angioödeme durch ACE-Hemmer und AT1Rezeptorblocker. Hautarzt 57:808-810. https:// doi.org/10.1007/s00105-005-1046-y

7. Ring JBK, Biedermann T et al (2014) Leitlinie zu Akuttherapie und Management der Anaphylaxie. Allergo JInt 23:96-112

8. Johansson SGO, BieberT, Dahl Retal (2004) Revised nomenclature for allergy for global use: report of the Nomenclature Review Committee of the World Allergy Organization, October 2003. J Allergy Clin Immunol 113:832-836

9. Wüthrich B (2012) Angioödeme; selten allergisch bedingt. Schweiz Med Forum 12(7):138-143
10. Levy J, RivardGE, WagnerEet al (2014) Examination of genetic variants involved in generation and biodisposition of kinins in patients with angioedema. Allergy Asthma Clin Immunol 10:60

11. Bezalel S, Mahlab-Guri K, Asher I et al (2015) Angiotensin-converting enzyme inhibitor-induced angioedema. Am J Med 128:120-125

12. Zuberbier T, Aberer W, Asero R et al (2014) The EAACI/GA2LEN/EDF/WAO guideline for the definition, classification, diagnosis, and management of urticaria: the 2013 revision and update. Allergy 69:868-887

13. Craig TJ, Bernstein JA, Farkas $H$ et al (2014) Diagnosis and treatment of Bradykinin-mediated Angioedema: outcomes from an Angioedema expert consensus meeting. Int Arch Allergy Immunol 165:119-127

14. Kanani A, Schellenberg R, Warrington R (2011) Urticaria and angioedema. Allergy Asthma Clin Immunol 7(Suppl 1):S9

15. Scalese M, Reinaker Ts (2016) Pharmacologic management of angioedema induced by angiotensin-converting enzyme inhibitors. Am J Health Syst Pharm 73:873

16. Bouillet L, Boccon-Gibod I, Berard F et al (2014) Recurrent angioedema: diagnosis strategy and biological aspects. Eur J Dermatol 24:293-296

17. Faisant C, Armengol G, Bouillet $L$ et al (2016) Angioedema triggered by medication blocking the Renin/Angiotensin system: retrospective study using the French National Pharmacovigilance Database. JClin Immunol 36:95-102

18. Cichon S, Martin L, c HH et al (2006) Increased activity of coagulation factor XII (Hageman factor) causes hereditary angioedema type III. Am J Hum Genet 79:1098-1104

19. Dewald G, BorkK (2006) Missense mutations in the coagulation factor XII (Hageman factor) gene in hereditary angioedema with normal C1 inhibitor. Biochem Biophys Res Commun 343:1286-1289

20. Stieber C, Cichon S, Magerl M et al (2017) Clinical utility gene card for hereditary angioedema with normal C1 inhibitor (HAenC1). Eur J Hum Genet 25:e1-e4. https://doi.org/10.1038/ejhg.2017.104

21. Bas M(2017) Theangiotensin-converting-enzymeinduced angioedema. Immunol Allergy Clin North Am 37:183-200

22. Bork K (2010) Rezidivierende Angioödeme mit potenzieller Erstickungsgefahr. Dtsch Arztebl Int 107:408

23. Hoover T, Lippmann M, Grouzmann E et al (2010) Angiotensin converting enzyme inhibitor induced angio-oedema: a review of the pathophysiology and risk factors. Clin Exp Allergy 40:50-61

24. Kaplan AP (2011) Drug-induced angioedema. J Angioedema 1:1422

25. Byrd JB, Touzin K, Sile S et al (2008) Dipeptidyl peptidase IV in Angiotensin-converting enzyme inhibitor - associated angioedema. Hypertension 51:141-147

26. Campbell DJ (2001) The Kallikrein - Kinin system in humans. Clin Exp Pharmacol Physiol 28:1060-1065

27. Scott SI, Andersen MF, Aagaard Let al (2017) Dipeptidyl peptidase-4 inhibitor induced angioedemaan overlooked and potentially lethal adverse drug reaction? Curr Diabetes Rev. https://doi.org/10. 2174/1573399813666170214113856

28. Jean M, Gera L, Charest-Morin X et al (2015) In vivo effects of Bradykinin $B(2)$ receptor agonists with varying susceptibility to Peptidases. Front Pharmacol 6:306

29. La Corte CAL, Carter AM, Rice Gl et al (2011) A functional XPNPEP2 promoter haplotype leads to reduced plasma aminopeptidase $P$ and increased 
risk of ACE inhibitor-induced angioedema. Hum Mutat 32:1326-1331

30. Lefebvre J, Murphey LJ, Hartert TV et al (2002) Dipeptidyl peptidase IV activity in patients with ACEinhibitor-associated angioedema. Hypertension 39:460-464

31. Duan QL, Nikpoor B, Dube MP et al (2005) A variant in XPNPEP2 is associated with angioedema induced by angiotensin I-converting enzyme inhibitors. Am J Hum Genet 77:617-626

32. Kuoppala A, Lindstedt KA, Saarinen J et al (2000) Inactivation of bradykinin by angiotensinconverting enzyme and by carboxypeptidase $\mathrm{N}$ in human plasma. Am J Physiol Heart Circ Physiol 278:H1069-H1074

33. Baram M, Kommuri A, Sellers SA et al (2013) ACE inhibitor-induced angioedema. J Allergy Clin Immunol Pract 1:442-445

34. Brown NJ, Byiers S, Carr D et al (2009) Dipeptidyl peptidase-IV inhibitor use associated with increased risk of ACE inhibitor-associated angioedema. Hypertension 54:516-523

35. Mashaghi A, Marmalidou A, Tehrani $M$ et al (2016) Neuropeptide substance $P$ and the immune response. Cell Mol Life Sci 73:4249-4264

36. Miller DR, Oliveria SA, Berlowitz DR et al (2008) Angioedema incidence in US veterans initiating angiotensin-converting enzyme inhibitors. Hypertension 51:1624-1630

37. Campo PF, Tahía D, Canto G, Mayorga C (2013) Angioedema induced by angiotensin-converting enzyme inhibitors. Curr Opin Allergy Clin Immuno 13:337

38. Toh S, Reichman ME, Houstoun M et al (2012) Comparative risk for angioedema associated with the use of drugs that target the reninangiotensin-aldosterone system. Arch Intern Med 172:1582-1589

39. Byrd JB, Adam A, Brown NJ (2006) Angiotensinconverting enzyme inhibitor-associated angioedema. Immunol Allergy Clin North Am 26:725-737

40. StauberTC-CR, Goldberg A (2014) Life-threatening angioedema induced by angiotensin-converting enzyme inhibitors: characteristics and risk factors. Am J Rhinol Allergy 28:54

41. Messerli FH, Nussberger J (2000) Vasopeptidase inhibition and angio-oedema. Lancet 356:608-609

42. Bas M, Greve J, Strassen U et al (2015) Angioedema induced by cardiovascular drugs: new players join old friends. Allergy 70:1196-1200

43. Nussberger J, Cugno M, Amstutz C et al (1998) Plasma bradykinin in angio-oedema. Lancet 351:1693-1697

44. Blais C Jr., Rouleau JL, Brown NJ et al (1999) Serum metabolism of bradykinin and des-Arg9bradykinin in patients with angiotensin-converting enzyme inhibitor-associated angioedema. Immunopharmacology 43:293-302

45. Adam A, Cugno M, Molinaro G et al (2002) Aminopeptidase $P$ in individuals with a history of angio-oedema on ACE inhibitors. Lancet 359:2088-2089

46. Molinaro G, Cugno M, Perez M et al (2002) Angiotensin-converting enzyme inhibitor-associated angioedema is characterized by a slower degradation of des-arginine ${ }^{9}$-Bradykinin. J Pharmacol Exp Ther 303:232-237

47. Pare G, Kubo M, Byrd JB et al (2013) Genetic variants associated with angiotensin-converting enzyme inhibitor-associated angioedema. Pharmacogenet Genomics 23:470-478

48. Woodard-Grice AV, Lucisano AC, Byrd JB et al (2010) Sex-dependent and race-dependent association of XPNPEP2 C-2399 A polymorphism with angiotensin-converting enzyme inhibitor-associated angioedema. Pharmacogenet Genomics 20:532-536

49. Brown NJ, Ray WA, Snowden M et al (1996) Black Americans have an increased rate of angiotensin converting enzyme inhibitor-associated angioedema. Clin Pharmacol Ther 60:8-13

50. Kostis JB, Kim HJ, Rusnak J et al (2005) Incidence and characteristics of angioedema associated with enalapril. Arch Intern Med 165:1637-1642

51. Gallagher PE, Li P, Lenhart JR et al (1999) Estrogen regulation of angiotensin-converting enzyme mRNA. Hypertension 33:323-328

52. Morimoto T, Gandhi TK, Fiskio JM et al (2004) An evaluation of risk factors for adverse drug events associated with angiotensin-converting enzyme inhibitors. JEval Clin Pract 10:499-509

53. Hornig B, Kohler C, Schlink D et al (2003) AT1receptor antagonism improves endothelial function in coronary artery disease by a bradykinin/B2 receptor-dependent mechanism. Hypertension 41:1092-1095

54. Campbell DJ, Krum H, Esler MD (2005) Losartan increases bradykinin levels in hypertensive humans. Circulation 111:315-320

55. Beavers CJ, Dunn SP, Macaulay TE (2011) The role of angiotensin receptor blockers in patients with angiotensin-converting enzyme inhibitor-induced angioedema. Ann Pharmacother 45:520-524

56. Haymore BR, Yoon J, Mikita CP et al (2008) Risk of angioedema with angiotensin receptor blockers in patients with prior angioedema associated with angiotensin-converting enzyme inhibitors: a meta-analysis. Ann Allergy Asthma Immunol 101:495-499

57. Dalzell JR, Seed A, Berry C et al (2014) Effects of neutral endopeptidase (neprilysin) inhibition on the response to other vasoactive peptides in small human resistance arteries: studies with thiorphan and omapatrilat. Cardiovasc Ther 32:13-18

58. Sulpizio AC, Pullen MA, Edwards RMet al (2004) The effect of acute angiotensin-converting enzyme and neutral endopeptidase 24.11 inhibition on plasma extravasation in the rat. J Pharmacol Exp Ther 309:1141-1147

59. Hurford R, Rezvani S, Kreimei M et al (2015) Incidence, predictors and clinical characteristics of orolingual angio-oedema complicating thrombolysis with tissue plasminogen activator for ischaemic stroke. J Neurol Neurosurg Psychiatr 86:520-523

60. Hill MD, Lye T, Moss Het al (2003) Hemi-orolingual angioedema and ACE inhibition after alteplase treatment of stroke. Neurology 60:1525-1527

61. Lambeir AM, Durinx C, Scharpe S et al (2003) Dipeptidyl-peptidase IV from bench to bedside: an update on structural properties, functions, and clinical aspects of the enzyme DPP IV. Crit Rev Clin Lab Sci 40:209-294

62. Drucker DJ, Nauck MA (2006) The incretin system: glucagon-like peptide- 1 receptor agonists and dipeptidyl peptidase- 4 inhibitors in type 2 diabetes. Lancet 368:1696-1705

63. Remm F, Franz WM, Brenner C (2016) Gliptins and their target dipeptidyl peptidase 4: implications for the treatment of vascular disease. Eur Heart J Cardiovasc Pharmacother 2:185-193

64. Fantus D, Thomson AW (2015) Evolving perspectives of mTOR complexes in immunity and transplantation. Am J Transplant 15:891-902

65. Duerr M, Glander P, Diekmann F et al (2010) Increased incidence of angioedema with ACE inhibitors in combination with mTOR inhibitors in kidney transplant recipients. Clin J Am Soc Nephrol 5:703-708

66. Mahe E, Morelon E, Lechaton S et al (2007) Angioedema in renal transplant recipients on sirolimus. Dermatology (Basel) 214:205-209

67. Byrd JB, Woodard-Grice A, Stone E et al (2010) Association of angiotensin-converting enzyme inhibitor-associated angioedema with transplant and immunosuppressant use. Allergy 65:1381-1387

68. Schwabe UPD, Ludwig K-D, Klauber J (2016) Arzneiverordnungs-Report 2016. Springer, Berlin Heidelberg 